\title{
Article \\ Access Site Related Vascular Complications Following Percutaneous Cardiovascular Procedures
}

\author{
Johanna Hetrodt, Christiane Engelbertz (D), Katrin Gebauer, Jacqueline Stella, Matthias Meyborg, Eva Freisinger, \\ Holger Reinecke and Nasser Malyar*
}

\section{check for} updates

Citation: Hetrodt, J.; Engelbertz, C.; Gebauer, K.; Stella, J.; Meyborg, M.;

Freisinger, E.; Reinecke, H.; Malyar, N.

Access Site Related Vascular

Complications Following

Percutaneous Cardiovascular Procedures. J. Cardiovasc. Dev. Dis. 2021, 8, 136. https://doi.org/ $10.3390 /$ jcdd 8110136

Academic Editor: Krishnaraj Sinhji Rathod

Received: 20 September 2021

Accepted: 20 October 2021

Published: 22 October 2021

Publisher's Note: MDPI stays neutral with regard to jurisdictional claims in published maps and institutional affiliations.

Copyright: (c) 2021 by the authors. Licensee MDPI, Basel, Switzerland. This article is an open access article distributed under the terms and conditions of the Creative Commons Attribution (CC BY) license (https:// creativecommons.org/licenses/by/ $4.0 /)$.
Department of Cardiology I-Coronary and Peripheral Vascular Disease, Heart Failure, University Hospital Muenster, Cardiol, 48149 Muenster, Germany; j.hetrodt@icloud.com (J.H.); christianemaria.engelbertz@ukmuenster.de (C.E.); katrin.gebauer@ukmuenster.de (K.G.); jacqueline.stella@ukmuenster.de (J.S.); matthias.meyborg@ukmuenster.de (M.M.); eva.freisinger@ukmuenster.de (E.F.); holger.reinecke@ukmuenster.de (H.R.)

* Correspondence: nasser.malyar@ukmuenster.de; Tel.: +49-251-83-47688

Abstract: Vascular access site complications (ASC) are among the most frequent complications of percutaneous cardiovascular procedures (PCP) and are associated with adverse outcome and high resources utilization. In this prospective study, we investigated patients with postprocedural clinical suspicion of ASC evaluated by duplex ultrasound (DUS) for the presence of ASC. We assessed the incidence, in-hospital outcome, treatment of complications and predictors for ASC. Overall, 12,901 patients underwent PCP during a 40 months period. Of those, 2890 (22.4\%) patients had postprocedural clinical symptoms of ASC and were evaluated using DUS. An ASC was found in 206 of the DUS examined patients (corresponding to $7.1 \%$ of the 2890 DUS examined patients). In $6.7 \%$ of all valvular/TAVI procedures, an ASC was documented, while coronary, electrophysiological and peripheral PCP had a comparable and low rate of complications (1.2-1.5\%). Pseudoaneurysm (PSA) was the most frequent ASC (67.5\%), followed by arteriovenous fistula $(13.1 \%)$, hematoma $(7.8 \%)$ and others $(11.7 \%)$. Of all PSA, $84(60.4 \%)$ were treated surgically, $44(31.6 \%)$ by manual compression and $11(7.9 \%)$ conservatively. Three $(0.02 \%)$ patients died due to hemorrhagic shock. In conclusion, femoral ASC are rare in the current era of PCP with PSA being the leading type of ASC. Nonetheless, patients with predisposing risk factors and postprocedural suspicious clinical findings should undergo a DUS to early detect and mitigate ASC-associated outcome.

Keywords: percutaneous cardiovascular procedures; access site complications; femoral access; pseudoaneurysm; outcome

\section{Introduction}

Coronary, electrophysiological, peripheral and valvular diagnostic and interventional percutaneous cardiovascular procedures (PCP) constitute the cornerstone of invasive cardiovascular disease (CVD) management. Even though the radial artery access has proven to be associated with fewer complications [1], femoral access is still widely used, and it is particularly needed for high-risk procedures requiring large-bore sheaths [2,3]. Postprocedural hemostasis of femoral access site is usually achieved either by manual compression or, increasingly, by the use of vascular closure devices. Though rare, percutaneous arteriotomy for PCP can lead to serious access site complications (ASC), the most common of which are pseudoaneurysms (PSA), hematomas, arteriovenous fistulas (AVF), dissections and vascular closure device related local stenoses or occlusions. The consequences include prolonged hospital stay, increased patient morbidity and mortality and higher treatment costs [4].

The aim of this study was to determine the contemporary rate of ACS in symptomatic patients, potential predictors and outcome of puncture-site related vascular complications 
in patients who underwent diverse types of $\mathrm{PCP}$ for diagnosis and therapy of CVD at a tertiary cardiovascular center in Germany.

\section{Materials and Methods}

\subsection{Patient Selection and Clinical Assessment}

We prospectively evaluated all PCP conducted via the femoral access at the University Hospital Muenster, a tertiary cardiovascular center in Germany, from 09/2011 until 12/2014. Patients with symptoms and/or conspicuous clinical examination for an ASC (visible hematoma, swelling or murmur on auscultation at the puncture site) following PCP were screened per duplex-ultrasound (DUS) for detection of ASC such as PSA, AVF, hematoma and others, which include stenosis/occlusion or dissection of the accessed vessels, abscess and complication of closure device. All ASC were managed according to the standard of care as instructed in the standard operating procedures of the institution. If more than one ASC were detected, the most clinical relevant ASC as the leading type was noted.

Information regarding patients' medical history and previous medication were extracted from the medical records. Procedural data including the vascular access site, the type of procedure and periprocedural administration of medication were assessed from the PCP protocols.

Clinical and procedural factors were investigated for their predictive capabilities for the incidence of all ASC and, particularly, of PSA. The study was approved by the local ethical committee.

\subsection{Statistical Analysis}

Descriptive statistics was applied to all the collected data. Mean and standard deviation were calculated for continuous variables, and the absolute and the relative frequency in percentage for discrete variables. All continuous variables were tested for normal distribution.

In order to identify risk factors for the occurrence of PSA, an explorative logistic regression analysis was performed in which patient characteristics and clinical parameters considered to be particularly relevant were used as predictors. To identify parameters associated with need for surgical treatment of ASC also a logistic regression analysis was performed. A two-tailed $p$ value of less than 0.05 was considered significant. All analyses were performed using SPSS 25 software (IBM SPSS Statistics 25, Chicago, IL, USA).

\section{Results}

During a period of 3 years and 4 months, a total of 12,901 PCP conducted via femoral access were performed, of which $6118(47.4 \%)$ were coronary angiographies/interventions, 3843 (29.8\%) electrophysiological testing/ablation, 2474 (19.2\%) peripheral vascular angiographies/interventions and $466(3.6 \%)$ valvular procedures, mainly transcatheter aortic valve implantation (TAVI) (Table 1). Due to symptoms and/or conspicuous findings during inspection and auscultation following PCP, $2890(22.4 \%)$ patients were evaluated per DUS for detection of any ASC. In 206 (7.1\% of DUS examined patients, corresponding 1.6\% of the whole study population) an ASC was found, of which 139 (67.5\%) were PSA, 27 (13.1\%) AVF, $16(7.8 \%)$ hematoma and 24 (11.7\%) other complications (Figure 1).

The frequencies of the different cardiovascular procedures, the number of the corresponding ASC and of the complications for each procedure type are illustrated in Table 1.

The rate of ASC following PCP was highest for valvular/TAVI procedures (6.7\%). Coronary, electrophysiological and peripheral PCP had a comparable and low rate of ASC (1.2-1.5\%). 
Table 1. Percutaneous cardiovascular procedures and corresponding access site complication according to procedure type.

\begin{tabular}{|c|c|c|c|}
\hline & $\begin{array}{c}\text { PCP } \\
n=12,901\end{array}$ & $\begin{array}{c}\text { Clinical Suspicion of } \\
\text { ASC } \\
n=2890\end{array}$ & $\begin{array}{c}\text { Confirmed ASC } \\
\qquad n=206\end{array}$ \\
\hline Coronary intervention, $n(\%)$ & $6118(47.4)$ & $1277(44.2)$ & $\begin{array}{c}92 \\
\text { (1.5\% of all coronary } \\
\text { interventions })\end{array}$ \\
\hline Peripheral intervention, $n(\%)$ & $2474(19.2)$ & $532(18.4)$ & $\begin{array}{c}37 \\
\text { (1.5\% of all peripheral } \\
\text { interventions) }\end{array}$ \\
\hline Electrophysiological intervention, $n(\%)$ & $3843(29.8)$ & $645(22.3)$ & $\begin{array}{c}46 \\
(1.2 \% \text { of all } \\
\text { electrophysiological } \\
\text { interventions })\end{array}$ \\
\hline TAVI/ valvular intervention, $n(\%)$ & $466(3.6)$ & $436(15.1)$ & $\begin{array}{c}31 \\
\begin{array}{c}\text { (6.7\% of all TAVI/valvular } \\
\text { interventions })\end{array}\end{array}$ \\
\hline
\end{tabular}

ASC denotes access site complication; PCP: percutaneous cardiovascular procedures; TAVI, transcatheter aortic valve implantation.

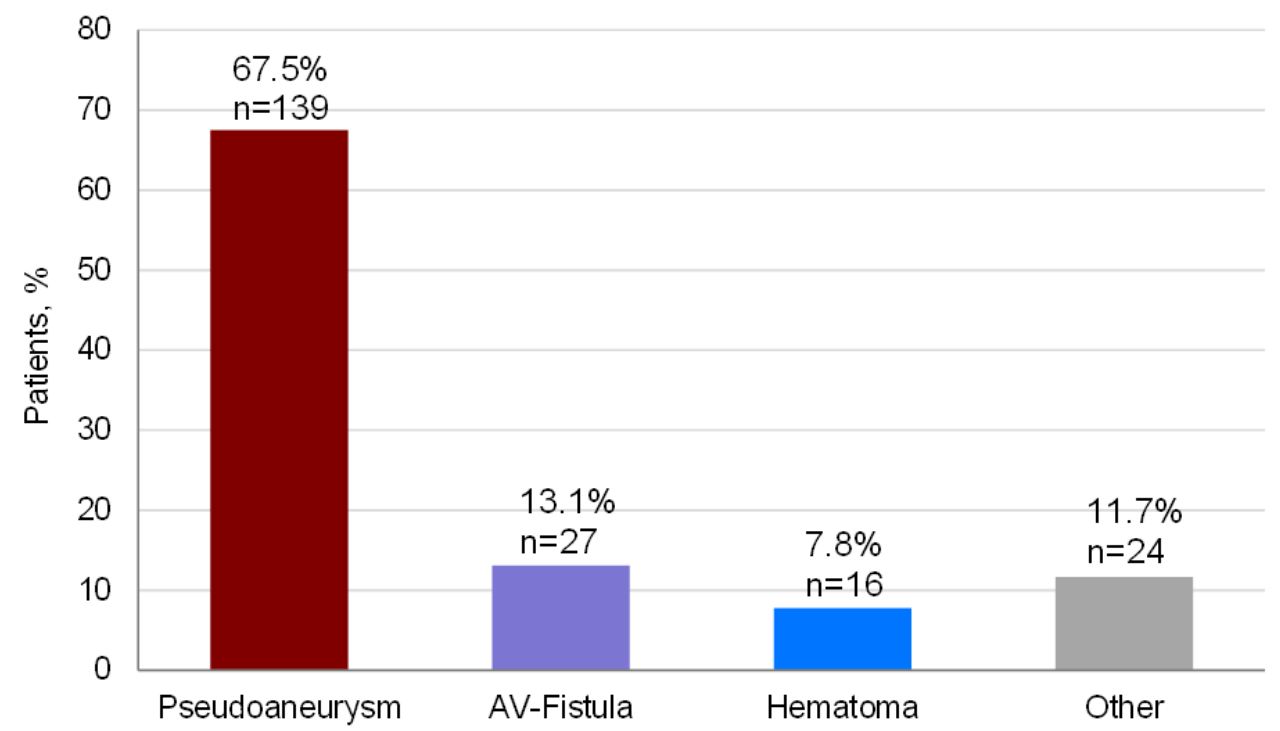

Figure 1. Type and frequency of access site complication. Pseudoaneurysm was the most frequent access site complication with $67.5 \%$ of all complications. Arteriovenous (AV) fistula, hematoma and other complications comprised between $7.8 \%$ and $13.1 \%$ of all access site complications. In patients with more than one access site complication, e.g., an AV fistula and a hematoma, only the most clinical relevant access site complication as the leading type was noted.

For patients with ASC, baseline characteristics and laboratory results at admission are presented in Table 2. Among all patients with ASC, 119 (57.8\%) were male, mean age was $71.8 \pm 12.7$ years and mean body mass index (BMI) was $26.8 \pm 5.0 \mathrm{~kg} / \mathrm{m}^{2}$. Coronary artery (CAD), peripheral artery (PAD) and cerebrovascular (CVD) disease were present in $104(50.5 \%), 47(22.8 \%)$ and 14 (6.8\%) of all patients, respectively. Renal insufficiency (eGFR $<60 \mathrm{~mL} / \mathrm{min} / 1.73 \mathrm{~m}^{2}$ ) was present in $84(41.8 \%)$ patients, and 5 $(2.4 \%)$ were on dialysis. 
Table 2. Baseline characteristics of patients with access site complications proven by duplex ultrasound.

\begin{tabular}{|c|c|}
\hline Characteristics & Total $(n=206)$ \\
\hline Age in years, mean $\pm \mathrm{SD}$ & $71.8 \pm 12.7$ \\
\hline $\mathrm{BMI}$ in $\mathrm{kg} / \mathrm{m}^{2}$, mean $\pm \mathrm{SD}$ & $26.8 \pm 5.0$ \\
\hline Male, $n(\%)$ & $119(57.8)$ \\
\hline \multicolumn{2}{|l|}{ Cardiovascular risk Factors and Comorbidities } \\
\hline Diabetes mellitus, $n(\%)$ & $44(21.4)$ \\
\hline Current smoker, $n(\%)$ & $29(14.1)$ \\
\hline Previous smoker, $n(\%)$ & $28(13.6)$ \\
\hline Dyslipidemia, $n(\%)$ & $71(34.5)$ \\
\hline Hypertension, $n(\%)$ & $139(67.5)$ \\
\hline CAD, $n(\%)$ & $104(50.5)$ \\
\hline $\mathrm{PAD}, n(\%)$ & $47(22.8)$ \\
\hline CVD, $n(\%)$ & $14(6.8)$ \\
\hline Renal insufficiency ${ }^{1}, n(\%)$ & $84(41.8)$ \\
\hline Renal replacement therapy, $(\%)$ & $5(2.4)$ \\
\hline \multicolumn{2}{|l|}{ Previous Medication } \\
\hline Acetylsalicylic acid $100 \mathrm{mg}, n(\%)$ & $70(34.0)$ \\
\hline Clopidogrel, $n(\%)$ & $136(66.0)$ \\
\hline Vitamin-K-Anatgonists, $n(\%)$ & $57(27.7)$ \\
\hline \multicolumn{2}{|l|}{ Laboratory Results } \\
\hline Creatinine in $\mathrm{mg} / \mathrm{dL}$, mean $\pm \mathrm{SD}$ & $1.27 \pm 1.16$ \\
\hline Glomerular filtration rate in $\mathrm{mL} / \mathrm{min} / 1.73 \mathrm{~m}^{2}$, mean $\pm \mathrm{SD}$ & $64.8 \pm 24.3$ \\
\hline Platelets in thousands $/ \mathrm{mL}$, mean $\pm \mathrm{SD}$ & $216 \pm 66$ \\
\hline International Normalized Ratio, mean \pm SD & $1.47 \pm 0.69$ \\
\hline Hemoglobin in $\mathrm{g} / \mathrm{dL}$, mean $\pm \mathrm{SD}$ & $12.9 \pm 1.9$ \\
\hline
\end{tabular}

${ }^{1}$ Renal insufficiency was defined as the current GFR $<60 \mathrm{~mL} / \mathrm{min} / 1.73 \mathrm{~m}^{2}$. Information about renal insufficiency and renal replacement therapy was available in 201 patients. BMI denotes body mass index; CAD: coronary artery disease; CHD: coronary heart disease; CVD: cerebrovascular disease; PAD: peripheral artery disease; SD: standard deviation.

\subsection{Procedural Characteristics of Patients with ASC}

Detailed procedural characteristics of patients with ASC are presented in Table 3. Right groin was the access site in most of the cases $(n=143 ; 69.4 \%)$ and the common femoral artery as the most frequently used access vessel $(n=137 ; 66.5 \%)$, followed by the femoral vein $(n=40 ; 19.4 \%)$, the superficial femoral artery $(n=22 ; 10.7 \%)$, the deep femoral artery $(n=2 ; 1 \%)$ and the common femoral artery and common femoral vein combined $(n=2 ; 1 \%)$. The majority of procedures $(n=182 ; 88.3 \%)$ were elective.

The procedures had a mean duration of $82 \pm 59 \mathrm{~min}$, mean sheath size was $6.9 \pm 3.1 \mathrm{FR}$, mean fluoroscopy time was $873 \pm 77 \mathrm{~s}$, with a mean radiation dose of $4248 \pm 4657 \mathrm{~Gy}$ and a contrast medium amount of $108 \pm 82 \mathrm{~mL}$. Vascular closure devices were used in $117(57.9 \%)$ of ASC cases, in 4 cases information concerning use of a closure device was missing. Angio-Seal ${ }^{\circledR}$ was the most frequent closure device type $(n=46 ; 39.3 \%)$.

\subsection{Type of Complication}

The type and frequencies of the ASC are illustrated in Figure 1. Among all ASC, PSA was the most frequent complication $(n=139 ; 67.5 \%)$, followed by AVF ( $n=27$; $13.1 \%)$ and local hematoma $(n=16 ; 7.8 \%)$. All other complications such as access-site stenosis/occlusion, local infection, persistent local pain, dysesthesia summed up to 24 $(11.7 \%)$ cases. During the study period, $3(0.02 \%)$ patients died due to hemorrhagic shock with massive retroperitoneal bleedings, all 3 undetected prior to lethal exit. 
Table 3. Procedural characteristics of patients with access site complications.

\begin{tabular}{|c|c|}
\hline Procedural Characteristics & Total $(n=206)$ \\
\hline \multicolumn{2}{|l|}{ Puncture site } \\
\hline Left, $n(\%)$ & $63(30.6)$ \\
\hline Right, $n(\%)$ & $143(69.4)$ \\
\hline \multicolumn{2}{|l|}{ Punctured vessel } \\
\hline Common femoral artery, $n(\%)$ & $137(66.5)$ \\
\hline Common femoral vein, $n(\%)$ & $40(19.4)$ \\
\hline Superficial femoral artery, $n(\%)$ & $22(10.7)$ \\
\hline Deep femoral artery, $n(\%)$ & $2(1.0)$ \\
\hline \multicolumn{2}{|l|}{ Indication $n(\%)$} \\
\hline Elective, $n(\%)$ & $182(88.3)$ \\
\hline Urgent, $n(\%)$ & $24(11.7)$ \\
\hline Sheath size in French, mean \pm SD & $6.9 \pm 3.1$ \\
\hline Vascular closure devices ${ }^{1}, n(\%)$ & $117(57.9)$ \\
\hline Angio-Seal ${ }^{\circledR}, n(\%)$ & $46(39.3)^{\#}$ \\
\hline FemoSeal $^{\mathrm{TM}}, n(\%)$ & $22(18.8)^{\#}$ \\
\hline StarClose $\mathrm{e}^{\mathrm{TM}}, n(\%)$ & $17(14.5)^{\#}$ \\
\hline Exoseal $^{\circledR}, n(\%)$ & $15(12.8)^{\#}$ \\
\hline Prostar ${ }^{\mathrm{TM}}, n(\%)$ & $15(12.8)^{\#}$ \\
\hline Examination duration in min, mean $\pm \mathrm{SD}$ & $82 \pm 59$ \\
\hline Fluoroscopy time in seconds, mean $\pm \mathrm{SD}$ & $873 \pm 77$ \\
\hline Radiation dose in Gy, mean \pm SD & $4248 \pm 4657$ \\
\hline Contrast medium in $\mathrm{mL}$, mean $\pm \mathrm{SD}$ & $108 \pm 82$ \\
\hline Hospital length of stay in days, mean $\pm \mathrm{SD}$ & $10 \pm 9$ \\
\hline Hemoglobin in $\mathrm{g} / \mathrm{dL}$, mean $\pm \mathrm{SD}$ & $12.9 \pm 1.9$ \\
\hline
\end{tabular}

${ }^{1}$ Information about type of vascular closure device was missing in two cases. " Percentage refers to 117 patients with vascular closure devices. SD denotes standard deviation.

\subsection{Postprocedural Management of Complications}

Mean hospital stay of patients with an ASC was $10 \pm 9$ days. Except for PSA, all other complications such as AV-fistula, hematoma, access-site stenosis, local infection, persistent local pain and dysesthesia were treated conservatively without any intervention. In patients with PSA $(n=139)$, the first manual compression therapy was successful in $22(15.8 \%)$ and a repeat manual compression the next day was successful in additional $22(15.8 \%)$ cases. Of the remaining patients with PSA and at least 2 unsuccessful manual compression attempts, $11(7.9 \%)$ were treated conservatively and surgical intervention was performed in $84(60.4 \%)$ of all cases.

\subsection{Outcome}

There was no sex-related tendency for specific ASC. Factors associated with need for surgical treatment of ASC were PSA (OR 24.72, 95\% confidence interval (CI) 7.82-78.18; $p<0.001$ ), use of vascular closure devices (OR 3.39, 95\% CI 1.61-7.16; $p=0.001$ ) and periprocedural use of acetylsalicylic acid (OR 3.29, 95\% CI 1.33-8.14; $p=0.01$, Figure 2a).

Regression analysis showed no significant effect characteristics such as sex, BMI, type of procedure, catheter size (in French), preprocedural international normalized ratio, and perioperative administration of heparin on the occurrence of PSA. Age and renal function (as indicated by the glomerular filtration rate), however, were significantly associated with postprocedural PSA (Figure $2 \mathrm{~b}$ ). Patients with postprocedural PSA were older (age $73.9 \pm 10.7$ years) than patients with other complications (67.5 \pm 15.4 years). Every year increase of life increased the risk for developing a PSA (OR 1.06, 95\% CI 1.03-1.10; $p<0.001$ ) and each unit decrease in eGFR increased the risk to 1.02 (OR 1.02, 95\% CI 1.00-1.04, $p=0.028$, Figure 2b). 
a Risk for need for surgical treatment of access site complication

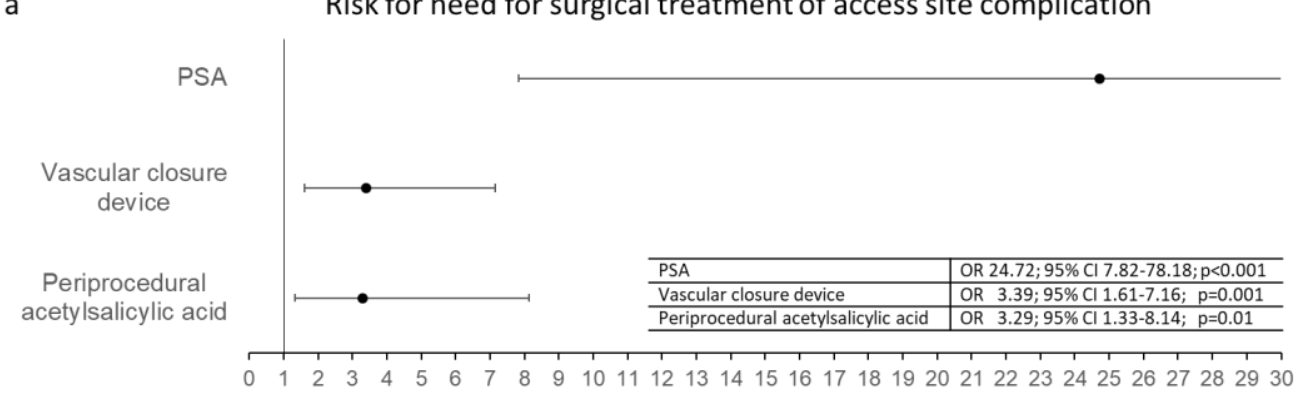

b Risk for development of a postprocedural pseudoaneurysm

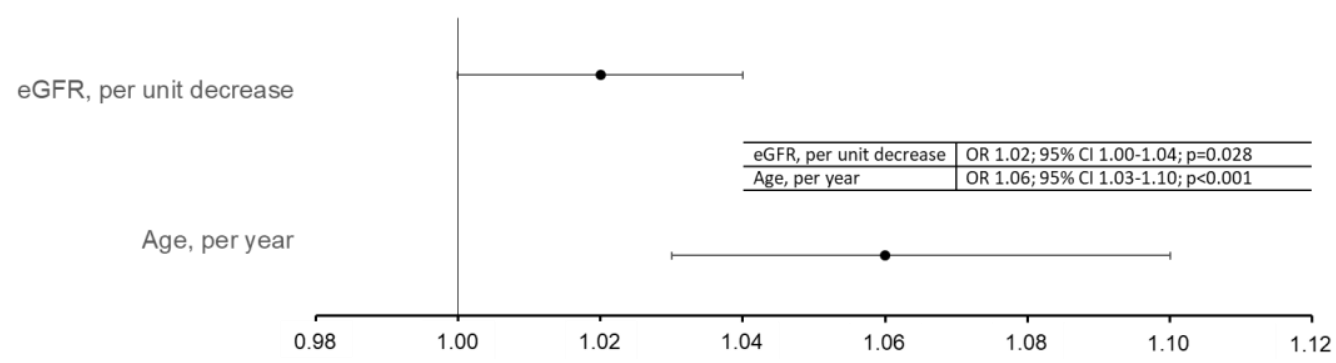

Figure 2. Forest plot showing risk factors for outcome. (a): Risk factors for need for surgical treatment of access site complication. Pseudoaneurysm (PSA), vascular closure device and periprocedural acetylsalicylic acid were identified as risk factors by logistic regression analysis. (b): Risk factors for the development of a postprocedural pseudoaneurysm Decreasing eGFR and increasing age were identified as risk factors by logistic regression analysis.

\section{Discussion}

\subsection{Procedure Types and Complication Rate}

Access site complications following PCP are among the most frequent periprocedural complications associated with adverse outcome and increased utilization of resource. In this prospective analysis of femoral ASC following diverse types of PCP at a tertiary cardiovascular center, we observed an overall complication rate of $1.6 \%$, which is lower than previously described [5]. The reported cumulative incidence for ASC in the literature varies, ranging from 0.05 to 21 per 100 interventions [6]. The variation is dependent on the intervention type, the population studied, the used case definition and on the use of diagnostic tools for detection of ASC [7-10]. In a meta-analysis of 30 studies, the rate of ASC was $1.5 \%$ to $9 \%$, of which $20 \%$ to $40 \%$ required surgical intervention at the access site [7]. Other studies indicated even a higher complication rate of up to 17\% [10]. A recent study performed by the University Hospital of Bonn, Germany, reports $1.9 \%$ of vascular complications following PCI [11]. Putting our data into perspective, the total PCP-related ASC-rate of $1.6 \%$ is lower than compared to the historical data, indicating a time- and experience-dependent decrease of ASC. However, it must be considered that we did not perform a systematic DUS-evaluation of all patients but only in those who had a clinically suspicious finding after the procedure. We might have missed some asymptomatic and clinical unapparent ASC, therefore underestimating the true burden of ASC following PCP. However, when considering the ASC rate in patients with clinical suspicious findings, the rate increased to $7.1 \%$, which is still within the range of previously published studies.

The highest rate of ASC was observed in patients undergoing TAVI. This is not surprising since TAVI patients are older and have more comorbidities including peripheral vascular disease, which are predisposing factors for ASC [5,12]. In the absence of a clear definition of ASC, previously published data about TAVI-associated ASC range between $6 \%$ and $36 \%$ [13].

Pseudoaneurysm was the most frequent ASC in our study group encompassing $67.5 \%$ of all ASC. In contrast to other ASC, most PSA require some kind of intervention, by either 
manual compression or surgical intervention. Another successful and minimal-invasive therapy is the injection of thrombin into the aneurysm sac. However, at the time of the study, this was not a routine approach at our institution. This explains the high fraction of surgical intervention following un-successful manual compression for the final treatment of PSA ( $n=84,60.4 \%$ ). The use of a closure device and periprocedural application of acetylsalicylic acid increased the risk for a subsequent surgical intervention by 3.39 and 3.29 , respectively.

In the literature, various predictors have been described to correlate with the occurrence of a PSA. A review from 2018 [14] found the following parameters to be associated with the incidence of postprocedural PSA: Advanced age, female sex, increased BMI, low platelet cell count, coronary heart disease, diabetes mellitus, arterial hypertension, hemodialysis, cardiovascular intervention, anticoagulative therapy, puncture of the left groin, catheter sizes $7-12 \mathrm{~F}$, too high/too low puncture and insufficient manual compression after puncture. In our exploratory study, however, only age and renal failure as indicated by reduced glomerular filtration rate were predictors of postprocedural incidence of PSA. Patients with preexisting renal failure/chronic kidney disease exhibit higher rates of general and specific access site complications. Moreover, the complication rate is related to the degree of renal impairment [15]. The impact of chronic renal insufficiency and outcomes after percutaneous coronary intervention was analyzed in a large-scale US Nationwide Inpatient Sample database ( $n=3187,404$ patients). The authors demonstrated that inpatients undergoing percutaneous coronary intervention, chronic renal insufficiency was a predictor of higher in-hospital mortality, higher periprocedural complications, longer hospital length of stay and higher costs. Though it is evident that patients with renal failure tend to higher rates of thrombotic events, they also are at higher risk for bleeding and hemostatic disorders such as pseudoaneurysm as well. Renal dysfunction/chronic kidney disease are associated with platelet dysfunction and abnormal soluble coagulation factors. However, the exact pathophysiological mechanisms are not fully understood yet.

This study demonstrates that PSA was not only the most common ASC $(67.5 \%$ of all complications) but also the most severe with a 24.7 times higher risk of vascular surgery intervention in the course compared to other complications.

Vascular closure devices have been shown to be associated with shorter time of hemostasis, rapid ambulation and fewer ASC compared to manual compression alone to achieve hemostasis at the puncture site [16]. However, as indicated by our data, if there is a PSA following the use of a closure device, the need for a surgical intervention is higher than without using a closure device.

\subsection{Limitations}

Only symptomatic and/or conspicuous patients were examined per DUS. It is most probable that we might have missed some asymptomatic patients with ASC and therefore underestimated the true burden of ASC. The data are derived from a single-center, therefore the generalizability is limited. The incidence and particularly the therapeutic management of ASC might be different in other centers, such as thrombin injection for treatment of PSA instead of surgical intervention.

\section{Conclusions}

The contemporary overall ASC rate following percutaneous cardiovascular procedures at a large-volume, tertiary cardiovascular center is with $1.6 \%$ at an acceptable low rate and as shown by historical data has decreased over the years probably due to extended experience of the treating physicians. Particularly patients with suspicious clinical findings after the procedures should be evaluated per DUS to confirm or exclude ASC. Pseudoaneurysms constitute two-third of all ASC and require frequently therapeutic interventions.

Author Contributions: Conceptualization, J.H. and N.M.; methodology, N.M., E.F., H.R.; formal analysis, J.H., C.E., N.M; investigation, M.M., N.M., K.G., J.S.; writing-original draft preparation, 
J.H.; writing-review and editing, J.H., N.M., C.E.; visualization, J.H., C.E.; supervision, N.M., E.F., H.R.; All authors have read and agreed to the published version of the manuscript.

Funding: This research received no external funding.

Institutional Review Board Statement: Ethical review and approval were waived for this study, due to pure observational nature of this study.

Informed Consent Statement: Informed consent was obtained from all subjects involved in the study.

Data Availability Statement: Data are stored at an electronic repository at the University Hospital Muenster.

Conflicts of Interest: Christiane Engelbertz has received travel support from Bayer Vital outside the submitted work. Katrin Gebauer reports travel support from Sanofi, personal fees from Bayer and NovoNordisk, and has been on the advisory board of Amgen, all outside the submitted work. Jacqueline Stella reports travel support from Daiichi Sankyo outside the submitted work. Eva Freisinger reports grants from Bayer and Pfizer outside the submitted work. Holger Reinecke reports personal fees from Bristol Myers Squibb, Daiichii Sankyo, DiaPlan, MedUpdate, NeoVasc, and Pfizer, all outside the submitted work. He acted as a consultant for NovoNordisk, Pluristem, and Pfizer. He took part in the conduction of multicentre trials of Bard, Bayer, Biotronik, Novartis, and Pluristem. Nasser Malyar reports personal fees from Bayer Vital, Bard, Cordis and Medtronic, all outside the frame of the submitted work. Johanna Hetrodt and Matthias Meyborg have nothing to declare.

\section{References}

1. Goel, S.; Pasam, R.T.; Raheja, H.; Gotesman, J.; Gidwani, U.; Ahuja, K.R.; Reed, G.; Puri, R.; Khatri, J.K.; Kapadia, S.R. Left main percutaneous coronary intervention-Radial versus femoral access: A systematic analysis. Catheter. Cardiovasc. Interv. 2020, 95, E201-E213.

2. Hamm, C.W.; Albrecht, A.; Bonzel, T.; Kelm, M.; Lange, H.; Schächinger, V.; Terres, W.; Voelker, W. Diagnostic heart catheterization. Clin. Res. Cardiol. 2008, 97, 475-512.

3. Kinnaird, T.; Anderson, R.; Gallagher, S.; Cockburn, J.; Sirker, A.; Ludman, P.; de Belder, M.; Copt, S.; Nolan, J.; Zaman, A.; et al. Vascular Access Site and Outcomes in 58,870 Patients Undergoing Percutaneous Coronary Intervention with a Previous History of Coronary Bypass Surgery: Results From the British Cardiovascular Interventions Society National Database. JACC Cardiovasc. Interv. 2018, 11, 482-492.

4. Ortiz, D.; Jahangir, A.; Singh, M.; Allaqaband, S.; Bajwa, T.K.; Mewissen, M.W. Access site complications after peripheral vascular interventions: Incidence, predictors, and outcomes. Circ. Cardiovasc. Interv. 2014, 7, 821-828.

5. Piper, W.D.; Malenka, D.J.; Ryan, T.J., Jr.; Shubrooks, S.J., Jr.; O'Connor, G.T.; Robb, J.F.; Farrell, K.L.; Corliss, M.S.; Hearne, M.J.; Kellett, M.A., Jr.; et al. Northern New England Cardiovascular Disease Study Group. Predicting vascular complications in percutaneous coronary interventions. Am. Heart J. 2003, 145, 1022-1029.

6. van Kesteren, F.; van Mourik, M.S.; Vendrik, J.; Wiegerinck, E.M.A.; Henriques, J.P.S.; Koch, K.T.; Wykrzykowska, J.J.; de Winter, R.J.; Piek, J.J.; van Lienden, K.P.; et al. Incidence, Predictors, and Impact of Vascular Complications After Transfemoral Transcatheter Aortic Valve Implantation with the SAPIEN 3 Prosthesis. Am. J. Cardiol. 2018, 121, 1231-1238.

7. Koreny, M.; Riedmüller, E.; Nikfardjam, M.; Siostrzonek, P.; Müllner, M. Arterial puncture closing devices compared with standard manual compression after cardiac catheterization: Systematic review and meta-analysis. JAMA 2004, 291, $350-357$.

8. Mlekusch, W.; Haumer, M.; Mlekusch, I.; Dick, P.; Steiner-Boeker, S.; Bartok, A.; Sabeti, S.; Exner, M.; Wagner, O.; Minar, E.; et al. Prediction of iatrogenic pseudoaneurysm after percutaneous endovascular procedures. Radiology 2006, 240, 597-602.

9. Etemad-Rezai, R.; Peck, D.J. Ultrasound-guided thrombin injection of femoral artery pseudoaneurysms. Can. Assoc. Radiol. J. 2003, 54, 118-120.

10. Bhatty, S.; Cooke, R.; Shetty, R.; Jovin, I.S. Femoral vascular access-site complications in the cardiac catheterization laboratory: Diagnosis and management. Interv. Cardiol. 2011, 3, 503-514.

11. Schahab, N.; Kavsur, R.; Mahn, T.; Schaefer, C.; Kania, A.; Fimmers, R.; Nickenig, G.; Zimmer, S. Endovascular management of femoral access-site and access-related vascular complications following percutaneous coronary interventions (PCI). PLoS ONE 2020, 15, e0230535.

12. Hoffmann, P.; Al-Ani, A.; von Lueder, T.; Hoffmann, J.; Majak, P.; Hagen, O.; Loose, H.; Kløw, N.E.; Opdahl, A. Access site complications after transfemoral aortic valve implantation-a comparison of Manta and ProGlide. CVIR Endovasc. $2018,1,20$.

13. Van Mieghem, N.M.; El Faquir, N.; Rahhab, Z.; Rodríguez-Olivares, R.; Wilschut, J.; Ouhlous, M.; Galema, T.W.; Geleijnse, M.L.; Kappetein, A.P.; Schipper, M.E.; et al. Incidence and predictors of debris embolizing to the brain during transcatheter aortic valve implantation. JACC Cardiovasc. Interv. 2015, 8, 718-724.

14. Stolt, M.; Braun-Dullaeus, R.; Herold, J. Do not underestimate the femoral pseudoaneurysm. Vasa 2018, 47, 177-185. 
15. Aziz, E.F.; Pulimi, S.; Coleman, C.; Florita, C.; Musat, D.; Tormey, D.; Fawzy, A.; Lee, S.; Herzog, E.; Coven, D.L.; et al. Increased vascular access complications in patients with renal dysfunction undergoing percutaneous coronary procedures using arteriotomy closure devices. J. Invasive Cardiol. 2010, 22, 8-13.

16. Smilowitz, N.R.; Kirtane, A.J.; Guiry, M.; Gray, W.A.; Dolcimascolo, P.; Querijero, M.; Echeverry, C.; Kalcheva, N.; Flores, B.; Singh, V.P.; et al. Practices and complications of vascular closure devices and manual compression in patients undergoing elective transfemoral coronary procedures. Am. J. Cardiol. 2012, 110, 177-182. 